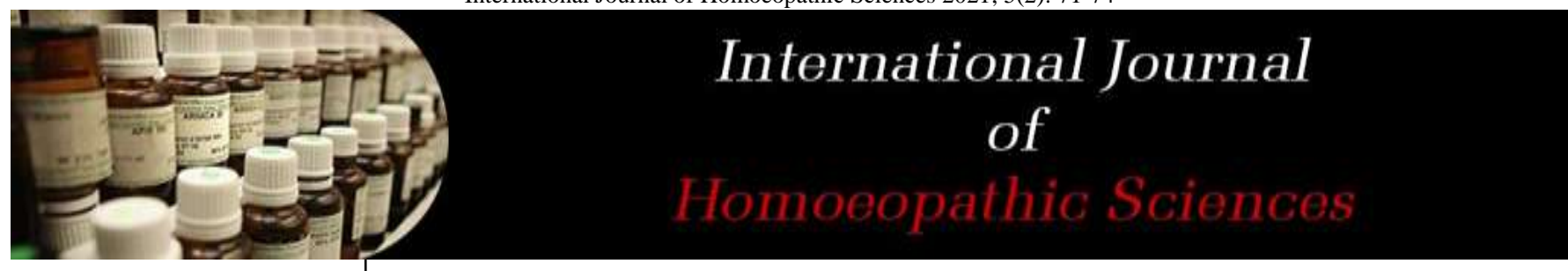

E-ISSN: 2616-4493 P-ISSN: 2616-4485 www.homoeopathicjournal.com IJHS 2021; 5(2): 71-74

Received: 02-01-2021

Accepted: 06-02-2021

Dr. Joseph T Kariyil MD (Homoeopathy), Junior Research Fellow, NHRIMH, Kottayam, Kerala, India

\section{A clinical study on homoeopathic medicines in pain management of haemorrhoids in adult age group}

\section{Dr. Joseph T Kariyil}

DOI: https://doi.org/10.33545/26164485.2021.v5.i2b.363

\section{Abstract}

Background and Objectives: Most of the treatment modes available for haemorrhoids are not up to the mark and a large number of patients are ultimately forced to undergo surgical interventions due to their symptoms. Pain is one of the common presentations of hemorrhoids and this study is aimed at understanding the improvement in pain of hemorrhoids in the adult age group using homoeopathic medicines.

Materials and Methods: Prospective, observational study was carried out in 30 patients by purposive sampling. Each case was followed for 6 months and outcome assessment was done by scores before and after study.

Result: Statistical analysis was done using a t-test where the result was t $(29)=24.80, p<0.05$ which shows there was a significant improvement of pain and improvement of pain in type $D$ patients is more correlated with the overall improvement in the population under study.

Keywords: Pain, haemorrhoids, homoeopathic medicines, adult age, type D personalities

\section{Introduction}

Haemorrhoids are the most common anorectal pathology affecting $70 \%$ of the adult population above thirty years of age is suffering from this disease ${ }^{[1]}$. Peak prevalence of haemorrhoids is noted between 45 and 65 years of age and the development of haemorrhoids before the age of 20 is unusual ${ }^{[2]}$. Haemorrhoids often show a hereditary pattern ${ }^{[3]}$ and the majority affected are females. But the actual cause of the haemorrhoidal disease is unknown [4].

According to the Journal of the Korean Society of Coloproctology, 5\% of the total population experience haemorrhoids at least once in their lifetimes. Besides, roughly $50 \%$ of the individuals in their 50's or older receive haemorrhoid treatments, and 10-20\% of the individuals who receive treatment require surgery which indirectly points towards the inefficiency of nonsurgical managements available ${ }^{[5]}$. Moreover surgical interventions like hemorrhoidal dearterialization and stapler hemorrhoidopexy ${ }^{[6]}$ have their complications.

Hemorrhoidal symptoms usually arise from enlarged internal hemorrhoids ${ }^{[7]}$. Common symptoms are bleeding, pain, mucus discharge, prolapsed and anal irritation ${ }^{[8]}$. Anoscopy is the most accurate method for examining the anal canal and the distal-most rectum. Kelley in his study found that anoscopy can identify $99 \%$ of anal lesions in subjects but colonoscopy revealed only $78 \%$ of anal lesions ${ }^{[9]}$.

There are many homoeopathic medicines capable of treating haemorrhoids [10]. Homoeopathic drugs are in very small doses which make them free from side effects ${ }^{[11]}$. In my practice, I have seen so many favourable clinical outcomes while treating haemorrhoids. Research conducted in a Homoeopathic hospital in West Bengal suggests haemorrhoids are treated with a success rate of 60.3 to $82.3 \%{ }^{[12]}$. There have been works on the effectiveness of individualized homoeopathic treatment in haemorrhoids ${ }^{[13]}$ but the area of acute pain management has been less explored. So I would like to add on and make the area complete. 


\section{Materials and Methods}

Patients suffering from pain due to hemorrhoids attending OPDs, IPDs and rural centers of Sarada Krishna Homoeopathic Medical College Hospital

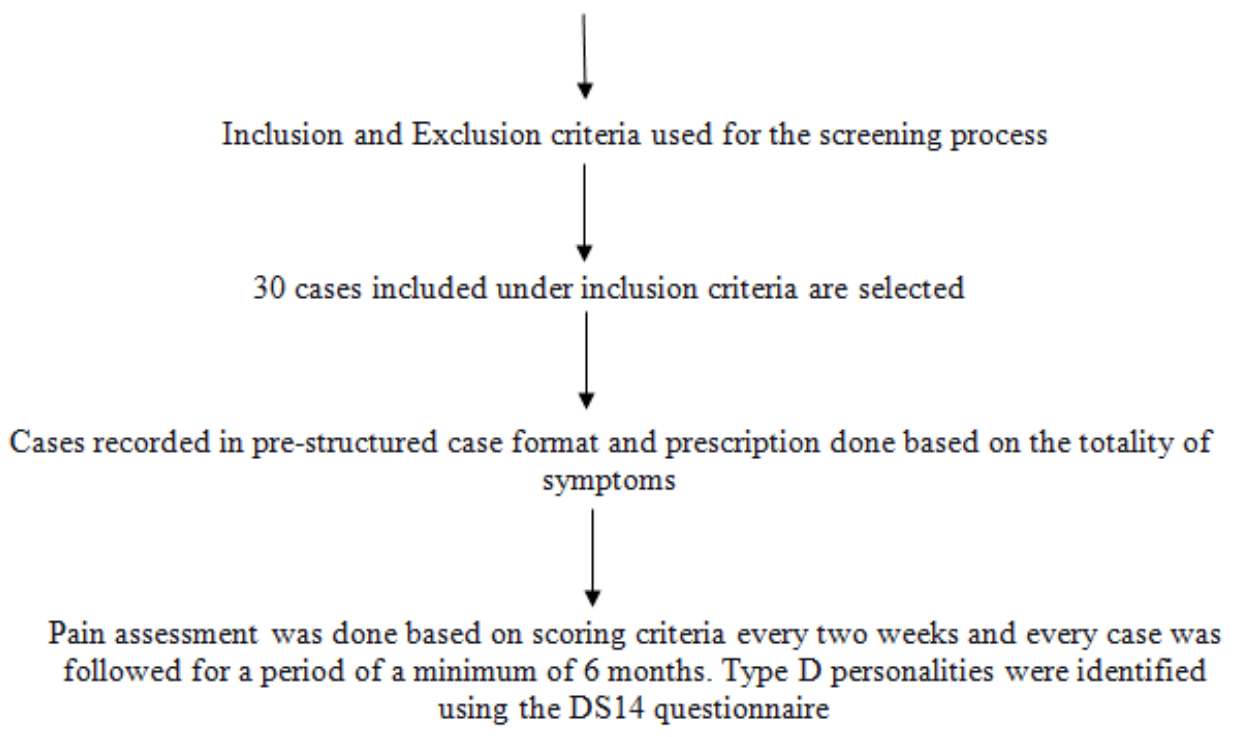

\subsection{Study design}

Observational, Non randomized, Prospective study was carried out.

\subsection{Study population}

30 cases who suffered from hemorrhoidal pain were purposively selected from OPD, IPD, and peripheral centers of Sarada Krishna Homoeopathic Medical College Hospital based upon inclusion as well as exclusion criteria.

\subsection{Inclusion criteria}

- Patient of adult age group - 18 to $60 \mathrm{yrs}$.

- Both sexes

- Based on symptomatology and protoscopic evaluation was done for confirmation

\subsection{Exclusion criteria}

Patients suffering from other diseases affecting anorectal region like fissure in ano, fistula in ano, anorectal abscess, malignancy, crohn's disease, ulcerative colitis, rectal polyp and other diseases having rectal complaints as secondary phenomena where excluded.

\subsection{Study period}

The study was conducted for a period of six months to one year.

\subsection{Method of collection of data}

Data were collected using a standardized case recording format.

\subsection{Prescription}

Based on totality of symptoms. Potency selection and dosage was based on homoeopathic principles.

\subsection{Follow up}

The review was done every two weeks and every case was followed for a period of a minimum of 6 months.

\subsection{Outcome assessment}

Improvement in pain is assessed before and after treatment based on scoring criteria. Pre-assessment is the baseline disease intensity of the study subjects. Post-assessment is the changes from the baseline condition of the study subjects.

\subsection{Statistical methods}

Paired t-test was conducted to conclude the study. The relationship of general pain score improvement with pain in type D personalities and non-type D personalities was assessed with correlation analysis.

\section{Results}

Thirty cases were subjected to study and all thirty cases showed improvement. Among them, three cases showed marked improvement, nineteen cases showed moderate improvement, and eight cases showed mild improvement. Statistical analysis was done using the t-test where the result was $\mathrm{t}(29)=24.80, p<0.05$ which shows there was a significant difference in pain before and after treatment. Sulphur was the most used remedy and the scale most used was centesimal. Eight medicines were used in treating these thirty cases. They are mentioned in descending frequency in which they have been prescribed: Sulphur 27\% $(n=8)$, Nux vom $20 \%(n=6)$, Lycopodium $17 \%(n=5)$, Nitric acid $13 \%(\mathrm{n}=4)$, Muriatic acid 10\% ( $\mathrm{n}=3)$, Nat mur $7 \%(\mathrm{n}=$ $2)$, Sepia $3 \%(\mathrm{n}=1)$ and Thuja $3 \%(\mathrm{n}=1)$. Centesimal potencies were prescribed most $77 \%(n=23)$, followed by decimal potencies $13 \%(n=4)$ and $10 \%(n=3)$ cases were prescribed in LM scale. On correlation analysis, the value of $\mathrm{R}$ on correlating total pain scores before and after the study was found to be 0.609 . This indicates a moderate positive correlation. The value of R2, the coefficient of determination, is 0.3709 and the regression line equation is $y=0.7417 x-2.4635$; whereas the value of $R$ for type $D$ patients were also 0.625 , showing moderate positive correlation; the correlation between pain scores of non-Type D patients was much lesser 0.4226 , than type $D$, which logically shows that the improvement pattern of type $D$ is more correlated and influences the overall correlation pattern in the population under study. 


\section{Discussion}

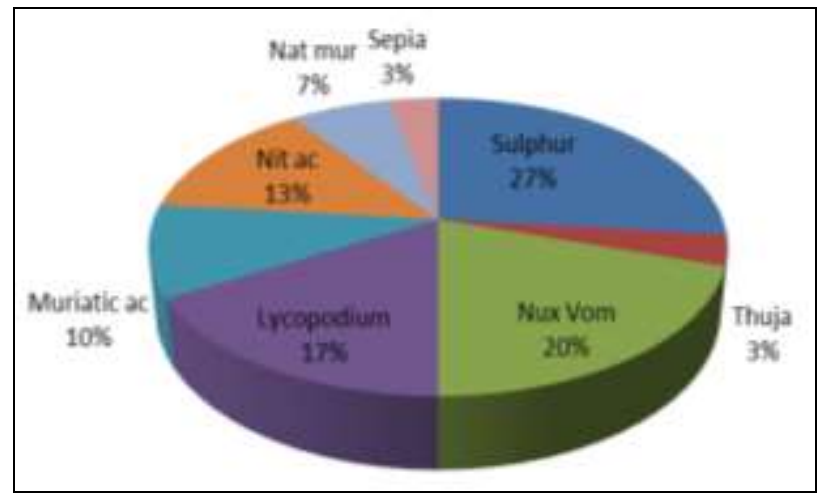

Fig 1: Distribution according to remedies

According to the study, there was a significant difference in the pain intensity scores after Homoeopathic treatment over a period of 6 months.

8 medicines were used for treating this condition (Fig 1) and overall $63.3 \%$ patients showed a moderate improvement as shown in Fig 2. Sulphur was given to the maximum number of patients (27\%) followed by Nux vomica (20\%). This is in accordance with an open observational pilot study and a multi-centric randomized single blind placebo controlled trial which had been done earlier ${ }^{[13,14]}$. So it must be re checked if Sulphur and Nux vom can be used as specific for haemorrhoid conditions. Allen's keynote says both remedies complements each other in all diseases so both remedies can be used another after other if the case demands. Guernsey frequently indicated four medicines also have Sulphur in them ${ }^{[15]}$.

According to the study, there was a significant difference in the pain intensity scores after Homoeopathic treatment for 6 months.

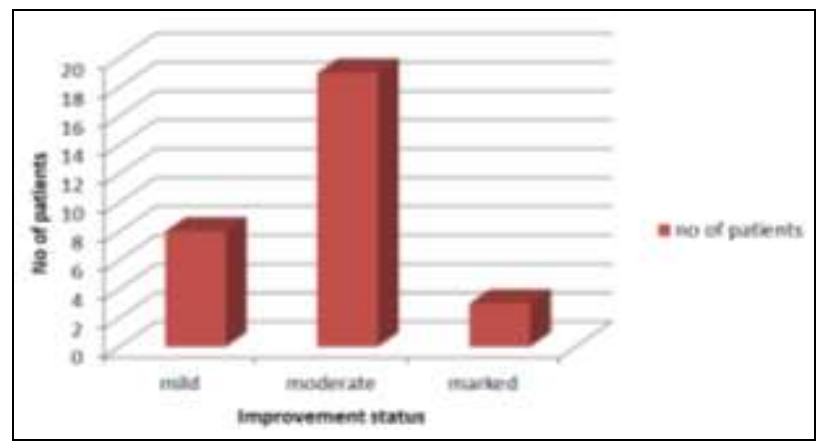

Fig 2: Distribution according to improvement status

$77 \%$ cases of this study also, centesimal potencies were prescribed (Fig 3) and LM scale was used least. This study result is also in accordance with the open observational study which has been done before in which centesimal scale was most commonly used ${ }^{[13]}$. A multi-centric randomized single-blind placebo-controlled trial has already shown the effect of LM potencies in acute attacks of haemorrhoid diseases ${ }^{[14]}$. So although LM potencies are used less in the current study, its role in haemorrhoids treatment can't be neglected.

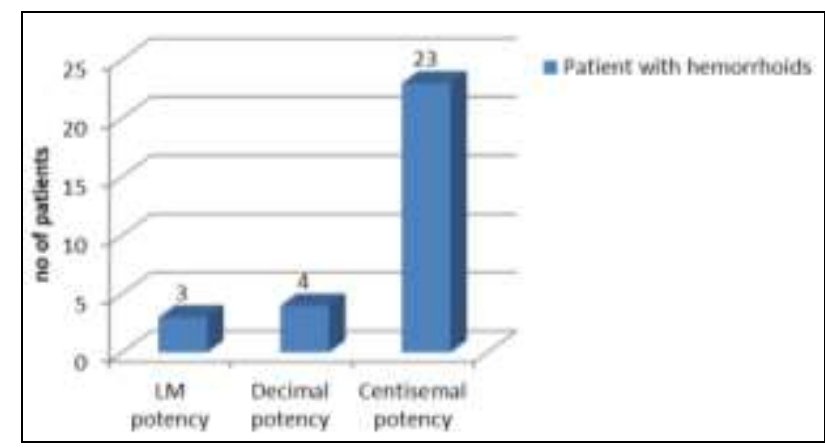

Fig 4: Distribution of cases according to potency

Type D personalities are people who showed more negative affectivity and social inhibition than the general population. They are the people who suffered more bodily sufferings compared to the general population ${ }^{[16]}$. Despite of more bodily sufferings; these people of the study experienced much improvement of pain than non-type D personality cases and the improvement pattern of type $\mathrm{D}$ is more correlated and influences the overall correlation pattern in the population under study (Fig 4, 5, 6). Such a result may have been aroused because the medicines prescribed were solely based upon a constitutional approach. This fact suggests that a homoeopathic medicine prescribed constitutionally can not only improve local pain of haemorrhoids but can influence the personality as a whole. I admit that such a suggestion needs more scientific correlations and request future researchers to address this issue also.

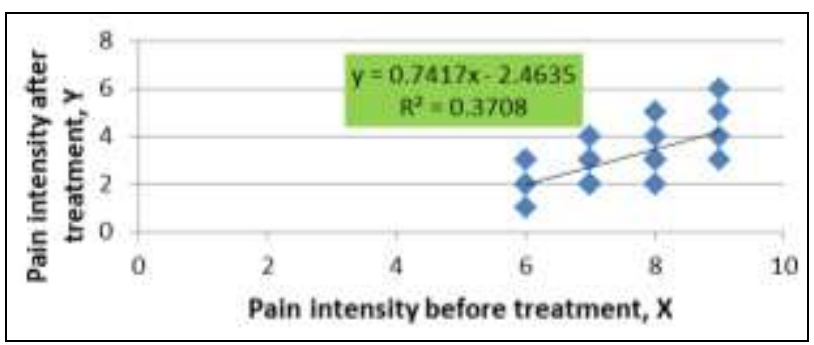

Fig 4: Scatter diagram showing correlation between pain intensities before and after treatment

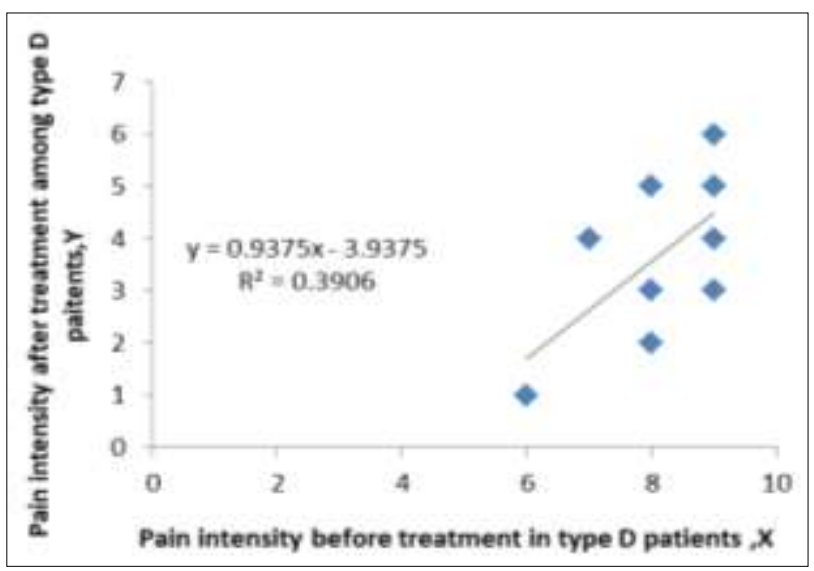

Fig 5: Scattered diagram showing correlation between pain intensities before and after treatment in Type D personalities 


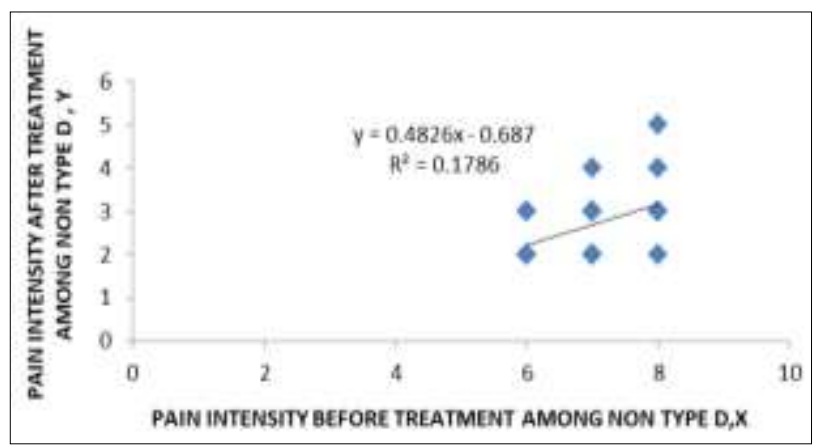

Fig 6: Scattered diagram showing correlation between pain intensities before and after treatment in non-type $\mathrm{D}$ personalities

\section{Conclusion}

Study done for 6 months illustrated there was considerable improvement in pain of haemorrhoids using homoeopathic treatment. Overall, results appeared convincing but a bigger sample size with an extended time of research would provide better results. The study would have been more scientific if the control (placebo) group would have been kept simultaneously to verify the effectiveness of treatment.

\section{Source of funding}

Nil

\section{Conflict of interest}

Nil

\section{References}

1. Sigh Sarabjith, Tayal Arun, Kaur Vineeth. Surgical treatment of haemorrhoids with stapler haemorrhoidopexy: our experience. JISMA 2011;24(2):97.

2. 15-Kaidar-Person Orit, Benjamin Person, Steven Wexner D. Hemorrhoidal Disease: A Comprehensive Review. Journal of the American College of Surgeons 204:1;102-17.

3. Warshaw JL, Turel R1. Occupational Aspects of Proctological Disease. New York State J Med 1997;57(18):3006-3010.

4. 11-Willis S, Junge K, Ebrahimi R, Prescher A, Schumpelick V. Haemorrhoids - a collagen disease. Colorectal Disease 2010;12(12):1249-1253.

5. Song, Seok-Gyu, Soung-Ho Kim. Optimal Treatment of Symptomatic Hemorrhoids. Journal of the Korean Society of Coloproctology 2011;27(6):277-81.

6. 4-Giamundo, Paolo. Advantages and Limits of Hemorrhoidal Dearterialization in the Treatment of Symptomatic Hemorrhoids. World Journal of Gastrointestinal Surgery 2016;8(1):1-4.

7. 21-Madoff Robert D, James Fleshman W. American Gastroenterological Association Technical Review on the Diagnosis and Treatment of Hemorrhoids. Gastroenterology 2004;126(5):1463-73.

8. 9-Williams Norman S, Christopher JK, O'Connell. Bailey and love's short practice of Surgery published by CRC Press 2008, 1253-1256.

9. 24-Kelly SM, Sanowski RA, Foutch PG et al. A prospective comparison of anoscopy and fiberendoscopy in detecting anal lesions. J Clin Gastroenterol 1986;8(6):658-660.

10. Kent JT. Repertory of the Hom. M.M., Published by B. Jain, New Delhi 2015, 619.
11. Khuda-Bukhsh, Anisur Rahman. Towards Understanding Molecular Mechanisms of Action of Homeopathic Drugs: An Overview. Molecular and Cellular Biochemistry 2003;253(1):339-45.

12. Saha Subhranil, Munmun Koley, Shubhamoy Ghosh, Mohan Giri, Asim Das, Rachna Goenka. Documentation of Prescriptions and Clinical Outcomes in a Homeopathic Hospital Setting in West Bengal, India. Journal of Evidence-Based Complementary \& Alternative Medicine 2015;20(3):180-85.

13. Das Kaushik Deb, Shubhamoy Ghosh, Asim Kumar Das, Aloke Ghosh, Ramkumar Mondal, Tanapa Banerjee et al. Reddy ES, Sharma PK, Raj PP. A clinical study on effect of Plantago in gingivitis by assessing bleeding and plaque index. Indian Journal of Research in Homoeopathy 2018;12(3):132-335-42.

14. Chakraborty PS, Varanasi R, Majumdar AK, Banoth K, Prasad S, Ghosh MS et al. Effect of Homoeopathic LM Potencies in Acute Attacks of Haemorrhoidal Disease: A Multicentric Randomized Single-Blind PlaceboControlled Trial. IJRH 2013;7(2):72-80.

15. Guernsey Jefferson WM. The homoeopathic therapeutics of haemorrhoids. Published by IBPP 2013, 7.

16. Sit, Mustafa, Edip Erdal Yilmaz, Fatih Canan, Osman Y1ldırım, Mehmet Mustafa Cetin. The Impact of Type D Personality on Health-Related Quality of Life in Patients with Symptomatic Haemorrhoids. Przeglad Gastroenterologiczny 2014;9(4):242-48. 